\title{
TRUNCATED EULER POLYNOMIALS
}

\author{
TAKAO KOMATSU* AND CLAUDIO DE J. PITA RUIZ V.**
}

\begin{abstract}
We define a truncated Euler polynomial $E_{m, n}(x)$ as a generalization of the classical Euler polynomial $E_{n}(x)$. In this paper we give its some properties and relations with the hypergeometric Bernoulli polynomial.
\end{abstract}

\section{INTRODUCTION}

For non-negative integer $m$, define truncated Euler polynomials $E_{m, n}(x)$ by

$$
\frac{\frac{2 t^{m}}{m !} e^{x t}}{e^{t}+1-\sum_{j=0}^{m-1} \frac{t^{j}}{j !}}=\sum_{n=0}^{\infty} E_{m, n}(x) \frac{t^{n}}{n !} .
$$

When $m=0, E_{n}(x)=E_{0, n}(x)$ is one of the definitions of Euler polynomials, given by

$$
\frac{2 e^{x t}}{e^{t}+1}=\sum_{n=0}^{\infty} E_{0, n}(x) \frac{t^{n}}{n !}
$$

When $x=0$ in (11), $E_{m, n}=E_{m, n}(0)$ are called truncated Euler numbers, given by

$$
\frac{\frac{2 t^{m}}{m !}}{e^{t}+1-\sum_{j=0}^{m-1} \frac{t^{j}}{j !}}=\sum_{n=0}^{\infty} E_{m, n} \frac{t^{n}}{n !} .
$$

Incomplete Bernoulli numbers [7] and incomplete Cauchy numbers [6, 8] are similar truncated numbers. Both of them are based upon the restricted and associated Stirling numbers of the second kind, and the restricted and associated Stirling numbers of the first kind. The restricted Bernoulli numbers $\mathfrak{B}_{n, \leq m}$ and the associated Bernoulli numbers $\mathfrak{B}_{n, \geq m}$ can be defined by

$$
\frac{\log \mathfrak{E}_{m}(-t)}{\mathfrak{E}_{m}(-t)-1}=\sum_{n=0}^{\infty} \mathfrak{B}_{n, \leq m} \frac{t^{n}}{n !}
$$

1991 Mathematics Subject Classification. Primary 11B68; Secondary 11B83, 11B37, 05A15, 05A19.

Key words and phrases. Euler polynomials, Truncated Euler polynomials, Bernoulli polynomials, Hypergeometric Bernoulli polynomials.

The first author was supported in part by the grant of Wuhan University and by the grant of Hubei Provincial Experts Program. 
and

$$
\frac{\log \left(1+e^{-t}-\mathfrak{E}_{m-1}(-t)\right)}{e^{-t}-\mathfrak{E}_{m-1}(-t)}=\sum_{n=0}^{\infty} \mathfrak{B}_{n, \geq m} \frac{t^{n}}{n !},
$$

respectively, where

$$
\mathfrak{E}_{m}(t)=\sum_{n=0}^{m} \frac{t^{n}}{n} .
$$

When $m \rightarrow \infty$ in the former case or $m=1$ in the latter case, we have the generating function of the classical Bernoulli numbers $\mathfrak{B}_{n}\left(\right.$ with $\left.\mathfrak{B}_{1}=1 / 2\right)$, defined by

$$
\frac{t}{1-e^{-t}}=\sum_{n=0}^{\infty} \mathfrak{B}_{n} \frac{t^{n}}{n !}
$$

Therefore, we have $\mathfrak{B}_{n}=\mathfrak{B}_{n, \leq \infty}=\mathfrak{B}_{n, \geq 1}$.

The restricted Cauchy numbers $\mathfrak{c}_{n, \leq m}$ and the associated Cauchy numbers $\mathfrak{c}_{n, \geq m}$ can be defined by

$$
\frac{e^{\mathfrak{F}_{m}(t)}-1}{\mathfrak{F}_{m}(t)}=\sum_{n=0}^{\infty} \mathfrak{c}_{n, \leq m} \frac{t^{n}}{n !}
$$

and

$$
\frac{e^{\log (1+t)-\mathfrak{F}_{m-1}(t)}-1}{\log (1+t)-\mathfrak{F}_{m-1}(t)}=\sum_{n=0}^{\infty} \mathfrak{c}_{n, \geq m} \frac{t^{n}}{n !},
$$

respectively, where

$$
\mathfrak{F}_{m}(t)=\sum_{n=1}^{m} \frac{(-1)^{n-1} t^{n}}{n}
$$

When $m \rightarrow \infty$ in the former case or $m=1$ in the latter case, we have the generating function of the classical Cauchy numbers $\mathfrak{c}_{n}$, defined by

$$
\frac{t}{\log (1+t)}=\sum_{n=0}^{\infty} \mathfrak{c}_{n} \frac{t^{n}}{n !}
$$

Therefore, we have $\mathfrak{c}_{n}=\mathfrak{c}_{n, \leq \infty}=\mathfrak{c}_{n, \geq 1}$.

A different type of generalization is based upon hypergeometric functions. For $N \geq 1$, define hypergeometric Bernoulli numbers $B_{N, n}$ (see [1, 2, 3]) by

$$
\frac{1}{{ }_{1} F_{1}(1 ; N+1 ; t)}=\frac{t^{N} / N !}{e^{t}-\sum_{n=0}^{N-1} t^{n} / n !}=\sum_{n=0}^{\infty} B_{N, n} \frac{t^{n}}{n !},
$$

where

$$
{ }_{1} F_{1}(a ; b ; z)=\sum_{n=0}^{\infty} \frac{(a)^{(n)}}{(b)^{(n)}} \frac{z^{n}}{n !}
$$


is the confluent hypergeometric function with $(x)^{(n)}=x(x+1) \cdots(x+n-1)$ $(n \geq 1)$ and $(x)^{(0)}=1$. When $N=1, B_{n}=B_{1, n}$ are classical Bernoulli numbers (with $B_{1}=-1 / 2$ ) defined by

$$
\frac{t}{e^{t}-1}=\sum_{n=0}^{\infty} B_{n} \frac{t^{n}}{n !}
$$

In addition, define hypergeometric Cauchy numbers $c_{N, n}$ (see [5]) by

$$
\frac{1}{{ }_{2} F_{1}(1, N ; N+1 ;-t)}=\frac{(-1)^{N-1} t^{N} / N}{\log (1+t)-\sum_{n=1}^{N-1}(-1)^{n-1} t^{n} / n}=\sum_{n=0}^{\infty} c_{N, n} \frac{t^{n}}{n !},
$$

where

$$
{ }_{2} F_{1}(a, b ; c ; z)=\sum_{n=0}^{\infty} \frac{(a)^{(n)}(b)^{(n)}}{(c)^{(n)}} \frac{z^{n}}{n !}
$$

is the Gauss hypergeometric function. When $N=1, \mathfrak{c}_{n}=c_{1, n}$ are classical Cauchy numbers defined by

$$
\frac{t}{\log (1+t)}=\sum_{n=0}^{\infty} \mathfrak{c}_{n} \frac{t^{n}}{n !}
$$

Furthermore, the hypergeometric Bernoulli polynomials [1, 2] are defined by

$$
\frac{\frac{t^{m}}{m !} e^{x t}}{e^{t}-\sum_{j=0}^{m-1} \frac{t^{j}}{j !}}=\sum_{n=0}^{\infty} B_{m, n}(x) \frac{t^{n}}{n !}
$$

or

$$
\frac{e^{x t}}{{ }_{1} F_{1}(1 ; m+1 ; t)}=\sum_{n=0}^{\infty} B_{m, n}(x) \frac{t^{n}}{n !},
$$

and the hypergeometric Cauchy polynomials $c_{M, N, n}(x)$ [5] are defined by

$$
\frac{1}{(1+t)^{x}} \frac{1}{{ }_{2} F_{1}(M, N ; N+1 ;-t)}=\sum_{n=0}^{\infty} c_{M, N, n}(x) \frac{t^{n}}{n !} .
$$

Observe that in the case $m=0$, formula (3) becomes

$$
e^{(x-1) t}=\sum_{n=0}^{\infty} B_{0, n}(x) \frac{t^{n}}{n !},
$$

from where we see that $B_{0, n}(x)=(x-1)^{n}$.

When $m=1$, then $B_{n}(x)=B_{1, n}(x)$ are the classical Bernoulli polynomials, defined by

$$
\frac{t e^{x t}}{e^{t}-1}=\sum_{n=0}^{\infty} B_{n}(x) \frac{t^{n}}{n !},
$$


and $c_{n}(x)=c_{1, n}(x)$ are the classical Cauchy polynomials, defined by

$$
\frac{t}{(1+t)^{x} \log (1+t)}=\sum_{n=0}^{\infty} c_{n}(x) \frac{t^{n}}{n !}
$$

In this paper, we give some properties of truncated Euler polynomials.

\section{Some PRoperties of truncated Euler polynomials}

Theorem 1. For $n \geq 1$, we have

$$
E_{1, n}(x)=2 n(x-1)^{n-1} .
$$

Proof. When $m=1$, (10) becomes

$$
\begin{aligned}
\sum_{n=1}^{\infty} E_{1, n}(x) \frac{t^{n-1}}{n !} & =2 e^{(x-1) t} \\
& =2 n \sum_{n=1}^{\infty} \frac{(x-1)^{n-1} t^{n-1}}{n !} .
\end{aligned}
$$

Comparing the coefficients on both sides, we get the result.

Theorem 2. We have

$$
E_{m, n}(x)=0 \quad(n=0,1, \ldots, m-1)
$$

and

$$
E_{m, n+m}(x)=2\left(\begin{array}{c}
n+m \\
n
\end{array}\right) x^{n}-\sum_{j=0}^{n}\left(\begin{array}{c}
n+m \\
j
\end{array}\right) E_{m, j}(x) \quad(n \geq 0) .
$$

Proof. From (11), we have

$$
\begin{aligned}
\frac{2 t^{m}}{m !} \sum_{n=0}^{\infty} \frac{(x t)^{n}}{n !} & =\left(\sum_{n=0}^{\infty} E_{m, n}(x) \frac{t^{n}}{n !}\right)\left(1+\sum_{j=m}^{\infty} \frac{t^{j}}{j !}\right) \\
& =\sum_{n=0}^{\infty} E_{m, n}(x) \frac{t^{n}}{n !}+\left(\sum_{n=0}^{\infty} E_{m, n}(x) \frac{t^{n}}{n !}\right)\left(\sum_{j=0}^{\infty} \frac{t^{j+m}}{(j+m) !}\right) .
\end{aligned}
$$


Hence,

$$
\begin{aligned}
& \left(\sum_{n=0}^{\infty} E_{m, n}(x) \frac{t^{n}}{n !}\right)\left(\sum_{j=0}^{\infty} \frac{t^{j+m}}{(j+m) !}\right) \\
& =\sum_{n=0}^{\infty} \frac{2 x^{n} t^{n+m}}{n ! m !}-\sum_{n=0}^{\infty} E_{m, n+m}(x) \frac{t^{n+m}}{(n+m) !}-\sum_{n=0}^{m-1} E_{m, n}(x) \frac{t^{n}}{n !} \\
& =\sum_{n=0}^{\infty}\left(2\left(\begin{array}{c}
n+m \\
m
\end{array}\right) x^{n}-E_{m, n+m}\right) \frac{t^{n+m}}{(n+m) !}-\sum_{n=0}^{m-1} E_{m, n}(x) \frac{t^{n}}{n !} .
\end{aligned}
$$

and

$$
\begin{aligned}
\left(\sum_{n=0}^{\infty} E_{m, n}(x) \frac{t^{n}}{n !}\right)\left(\sum_{j=0}^{\infty} \frac{t^{j+m}}{(j+m) !}\right) & =\sum_{n=0}^{\infty} \sum_{j=0}^{n} E_{m, j}(x) \frac{t^{j}}{j !} \frac{t^{n-j+m}}{(n-j+m) !} \\
& =\sum_{n=0}^{\infty}\left(\sum_{j=0}^{n}\left(\begin{array}{c}
n+m \\
j
\end{array}\right) E_{m, j}(x)\right) \frac{t^{n+m}}{(n+m) !} .
\end{aligned}
$$

Comparing the coefficients, we get the results.

Example. When $m=2$, we have $E_{2,0}(x)=E_{2,1}(x)=0$. From the recurrence relation

$$
E_{2, n+2}(x)=2\left(\begin{array}{c}
n+2 \\
2
\end{array}\right) x^{n}-\sum_{j=0}^{n}\left(\begin{array}{c}
n+2 \\
j
\end{array}\right) E_{2, j}(x),
$$

by putting $n=0,1,2$, we get

$$
\begin{aligned}
E_{2,2}(x) & =2, \\
E_{2,3}(x) & =6 x \\
E_{2,4}(x) & =12 x^{2}-\sum_{j=0}^{2}\left(\begin{array}{l}
4 \\
j
\end{array}\right) E_{2, j}(x) \\
& =12 x^{2}-\left(\begin{array}{l}
4 \\
2
\end{array}\right) \cdot 2=12\left(x^{2}-1\right),
\end{aligned}
$$

Similarly one gets $E_{2,5}(x)=20\left(x^{3}-3 x-1\right), E_{2,6}(x)=30(x+1)\left(x^{3}-5 x^{2}-\right.$ $x+9$ ), and so on.

\section{Theorem 3.}

$$
E_{m, n}(x+y)=\sum_{j=0}^{n}\left(\begin{array}{l}
n \\
j
\end{array}\right) E_{m, j}(x) y^{n-j} .
$$


Proof. From (11), we get

$$
\begin{aligned}
\sum_{n=0}^{\infty} E_{m, n}(x+y) \frac{t^{n}}{n !} & =\frac{\frac{2 t^{m}}{m !} e^{(x+y) t}}{e^{t}+1-\sum_{j=0}^{m-1} \frac{t^{j}}{j !}} \\
& =\frac{\frac{2 t^{m}}{m !} e^{x t}}{e^{t}+1-\sum_{j=0}^{m-1} \frac{t^{j}}{j !}} e^{y t} \\
& =\left(\sum_{n=0}^{\infty} E_{m, n}(x) \frac{t^{n}}{n !}\right)\left(\sum_{n=0}^{\infty} \frac{y^{n} t^{n}}{n !}\right) \\
& =\sum_{n=0}^{\infty} \sum_{j=0}^{n} E_{m, j}(x) \frac{t^{j}}{j !} \frac{y^{n-j} t^{n-j}}{(n-j) !} \\
& =\sum_{n=0}^{\infty}\left(\sum_{j=0}^{n}\left(\begin{array}{c}
n \\
j
\end{array}\right) E_{m, j}(x) y^{n-j}\right) \frac{t^{n}}{n !}
\end{aligned}
$$

Comparing the coefficients on both sides, we get the desired result.

Theorem 4. For $m, n \geq 0$, we have

$$
E_{m, n}(x)=\sum_{k=0}^{n}\left(\begin{array}{l}
n \\
k
\end{array}\right) E_{m, n-k} x^{k} .
$$

Proof. By the definition (1), we have

$$
\begin{aligned}
\sum_{n=0}^{\infty} E_{m, n}(x) \frac{t^{n}}{n !} & =\frac{\frac{2 t^{m}}{m !}}{e^{t}+1-\sum_{j=0}^{m-1} \frac{t^{j}}{j !}} e^{x t} \\
& =\left(\sum_{n=0}^{\infty} E_{m, n}(x) \frac{t^{n}}{n !}\right)\left(\sum_{n=0}^{\infty} x^{n} \frac{t^{n}}{n !}\right) \\
& =\sum_{n=0}^{\infty} \sum_{k=0}^{n}\left(\begin{array}{l}
n \\
k
\end{array}\right) E_{m, n-k} x^{k} \frac{t^{n}}{n !} .
\end{aligned}
$$

Comparing the coefficients of both sides, we get the desired result.

The generating funcion of the Stirling numbers of the second kind denoted by $\left\{\begin{array}{l}n \\ k\end{array}\right\}$ is given by

$$
\frac{\left.\left(e^{t}-1\right)^{k}\right)}{k !}=\sum_{n=0}^{\infty}\left\{\begin{array}{l}
n \\
k
\end{array}\right\} \frac{t^{n}}{n !} .
$$

The falling factorial $(x)_{n}$ and the rising factorial $(x)^{(n)}$ are defined by $(x)_{n}=$ $x(x-1) \cdots(x-n+1)$ and $(x)^{(n)}=x(x+1) \cdots(x+n-1)(n \geq 1)$ with $(x)_{0}=(x)^{(0)}=1$, respectively. 
Theorem 5. For $m, n \geq 0$, we have

$$
E_{m, n}(x)=\sum_{\mu=0}^{n} \sum_{l=\mu}^{n}\left\{\begin{array}{l}
l \\
\mu
\end{array}\right\}\left(\begin{array}{l}
n \\
l
\end{array}\right) E_{m, n-l} \cdot(x)_{\mu} .
$$

Proof. Since

$$
\sum_{\mu=0}^{l}\left\{\begin{array}{l}
l \\
\mu
\end{array}\right\}(x)_{\mu}=x^{l}
$$

By Theorem 4, we have

$$
\begin{aligned}
E_{m, n}(x) & =\sum_{l=0}^{n}\left(\begin{array}{l}
n \\
l
\end{array}\right) E_{m, n-l} x^{l} \\
& =\sum_{l=0}^{n}\left(\begin{array}{l}
n \\
l
\end{array}\right) E_{m, n-l} \sum_{\mu=0}^{l}\left\{\begin{array}{l}
l \\
\mu
\end{array}\right\}(x)_{\mu} \\
& =\sum_{\mu=0}^{n} \sum_{l=\mu}^{n}\left\{\begin{array}{l}
l \\
\mu
\end{array}\right\}\left(\begin{array}{l}
n \\
l
\end{array}\right) E_{m, n-l} \cdot(x)_{\mu} .
\end{aligned}
$$

Theorem 6. For $m, n \geq 0$, we have

$$
E_{m, n}(x)=\sum_{\mu=0}^{n} \sum_{l=\mu}^{n}\left\{\begin{array}{l}
l \\
\mu
\end{array}\right\}\left(\begin{array}{l}
n \\
l
\end{array}\right) E_{m, n-l}(-\mu) \cdot(x)^{(\mu)}
$$


Proof. We have

$$
\begin{aligned}
\sum_{n=0}^{\infty} E_{m, n}(x) \frac{t^{n}}{n !} & =\frac{\frac{2 t^{m}}{m !}}{e^{t}+1-\sum_{j=0}^{m-1} \frac{t^{j}}{j !}}\left(e^{-t}\right)^{-x} \\
& =\frac{\frac{2 t^{m}}{m !}}{e^{t}+1-\sum_{j=0}^{m-1} \frac{t^{j}}{j !}} \sum_{\mu=0}^{\infty}\left(\begin{array}{c}
x+\mu-1 \\
\mu
\end{array}\right)\left(1-e^{-t}\right)^{-x} \\
& =\frac{\frac{2 t^{m}}{m !}}{e^{t}+1-\sum_{j=0}^{m-1} \frac{t^{j}}{j !}} \sum_{\mu=0}^{\infty}(x)^{(\mu)} \frac{\left(e^{t}-1\right)^{\mu}}{\mu !} e^{-\mu t} \\
& =\sum_{\mu=0}^{\infty}(x)^{(\mu)}\left(\sum_{n=0}^{\infty}\left\{\begin{array}{l}
n \\
\mu
\end{array}\right\} \frac{t^{n}}{n !}\right)\left(\sum_{n=0}^{\infty} E_{m, n}(-\mu) \frac{t^{n}}{n !}\right) \\
& =\sum_{\mu=0}^{\infty}(x)^{(\mu)} \sum_{n=0}^{\infty}\left(\begin{array}{l}
n \\
l
\end{array}\right) \sum_{l=0}^{n}\left\{\begin{array}{l}
l \\
\mu
\end{array}\right\} E_{m, n-l}(-\mu) \frac{t^{n}}{n !} \\
& =\sum_{n=0}^{\infty} \sum_{\mu=0}^{n} \sum_{l=\mu}^{n}\left\{\begin{array}{l}
l \\
\mu
\end{array}\right\}\left(\begin{array}{l}
n \\
l
\end{array}\right) E_{m, n-l}(-\mu) \cdot(x)^{(\mu)} \frac{t^{n}}{n !}
\end{aligned}
$$

Comparing the coefficients, we get the desired result.

We finish this section by mentioning the relation with Frobenius-Euler polynomials. For $\lambda \in \mathbb{C}$ with $\lambda \neq 1$ and a nonnegative integer $r$, Frobenius-Euler polynomials $H_{n}^{(r)}(x \mid \lambda)$ are defined by

$$
\left(\frac{1-\lambda}{e^{t}-\lambda}\right)^{r} e^{x t}=\sum_{n=0}^{\infty} H_{n}^{(r)}(x \mid \lambda) \frac{t^{n}}{n !}
$$

(see e.g. [4]).

Theorem 7. For $m, n \geq 0$, we have

$$
E_{m, n}(x)=\sum_{\mu=0}^{n} \frac{1}{(1-\lambda)^{r}}\left(\begin{array}{l}
n \\
\mu
\end{array}\right) \sum_{i=0}^{r}\left(\begin{array}{l}
r \\
i
\end{array}\right)(-1)^{r-i} E_{m, n-\mu}(i) \cdot H_{\mu}^{(r)}(x \mid \lambda) .
$$


Proof. We have

$$
\begin{aligned}
& \sum_{n=0}^{\infty} E_{m, n}(x) \frac{t^{n}}{n !} \\
& =\frac{\frac{2 t^{m}}{m !}}{e^{t}+1-\sum_{j=0}^{m-1} \frac{t^{j}}{j !}}\left(\frac{e^{t}-\lambda}{1-\lambda}\right)^{r}\left(\frac{1-\lambda}{e^{t}-\lambda}\right)^{r} e^{x t} \\
& =\left(\sum_{n=0}^{\infty} E_{m, n} \frac{t^{n}}{n !}\right) \sum_{i=0}^{r}\left(\begin{array}{c}
r \\
i
\end{array}\right)\left(e^{t i}\right)(-\lambda)^{r-i} \frac{1}{(1-\lambda)^{r}}\left(\sum_{n=0}^{\infty} H_{n}^{(r)}(x \mid \lambda) \frac{t^{n}}{n !}\right) \\
& =\sum_{i=0}^{r}\left(\begin{array}{l}
r \\
i
\end{array}\right)(-\lambda)^{r-i} \frac{1}{(1-\lambda)^{r}}\left(e^{t i}\right) \sum_{n=0}^{\infty} \sum_{\mu=0}^{n}\left(\begin{array}{c}
n \\
\mu
\end{array}\right) E_{m, n-\mu} H_{\mu}^{(r)}(x \mid \lambda) \frac{t^{n}}{n !} \\
& =\sum_{n=0}^{\infty} \sum_{\mu=0}^{n} \frac{1}{(1-\lambda)^{r}}\left(\begin{array}{c}
n \\
\mu
\end{array}\right) \sum_{i=0}^{r}\left(\begin{array}{c}
r \\
i
\end{array}\right)(-1)^{r-i} E_{m, n-\mu}(i) \cdot H_{\mu}^{(r)}(x \mid \lambda) \frac{t^{n}}{n !} .
\end{aligned}
$$

Comparing the coefficients on both sides, we get the desired result.

\section{Relations With hypergeometric Bernoulli polynomials}

In this section, we shall show several relations with hypergeometric Bernoulli polynomials.

Theorem 8. For non-negative integers $n$ and $m$, we have

$$
\begin{aligned}
\left(\begin{array}{c}
n+m \\
n
\end{array}\right) \sum_{j=0}^{n}\left(\begin{array}{l}
n \\
j
\end{array}\right)\left(B_{m, j}(x) y^{n-j}\right. & \left.-\frac{1}{2} E_{m, j}(y) x^{n-j}\right) \\
& =\frac{1}{2} \sum_{j=0}^{n+m}\left(\begin{array}{c}
n+m \\
j
\end{array}\right) E_{m, j}(y) B_{m, n+m-j}(x) .
\end{aligned}
$$

Proof of Theorem 8. From (11), we have

$$
\begin{aligned}
\frac{z^{m}}{m !} e^{y t} & =\left(\sum_{n=0}^{\infty} \frac{1}{2} E_{m, n}(y) \frac{t^{n}}{n !}\right)\left(e^{t}-\sum_{j=0}^{m-1} \frac{t^{j}}{j !}+1\right) \\
& =\left(\sum_{n=0}^{\infty} \frac{1}{2} E_{m, n}(y) \frac{t^{n}}{n !}\right)\left(e^{t}-\sum_{j=0}^{m-1} \frac{t^{j}}{j !}\right)+\left(\sum_{n=0}^{\infty} \frac{1}{2} E_{m, n}(y) \frac{t^{n}}{n !}\right) .
\end{aligned}
$$


Hence,

$$
\begin{aligned}
& \frac{t^{m}}{m !} e^{y t} \sum_{n=0}^{\infty} B_{m, n}(x) \frac{t^{n}}{n !} \\
& =\left(\sum_{n=0}^{\infty} \frac{1}{2} E_{m, n}(y) \frac{t^{n}}{n !}\right) \frac{z^{m}}{m !} e^{x t}+\left(\sum_{n=0}^{\infty} \frac{1}{2} E_{m, n}(y) \frac{t^{n}}{n !}\right)\left(\sum_{n=0}^{\infty} B_{m, n}(x) \frac{t^{n}}{n !}\right)
\end{aligned}
$$

which can be written as

$$
\begin{aligned}
& \frac{t^{m}}{m !}\left(e^{y t} \sum_{n=0}^{\infty} B_{m, n}(x) \frac{t^{n}}{n !}-e^{x t} \sum_{n=0}^{\infty} \frac{1}{2} E_{m, n}(y) \frac{t^{n}}{n !}\right) \\
& =\left(\sum_{n=0}^{\infty} \frac{1}{2} E_{m, n}(y) \frac{t^{n}}{n !}\right)\left(\sum_{n=0}^{\infty} B_{m, n}(x) \frac{t^{n}}{n !}\right) .
\end{aligned}
$$

After the products, we get

$$
\begin{aligned}
& \frac{t^{m}}{m !} \sum_{n=0}^{\infty}\left(\sum_{j=0}^{n}\left(\begin{array}{l}
n \\
j
\end{array}\right)\left(B_{m, j}(x) y^{n-j}-\frac{1}{2} E_{m, j}(y) x^{n-j}\right)\right) \frac{t^{n}}{n !} \\
& =\sum_{n=0}^{\infty}\left(\sum_{j=0}^{n} \frac{1}{2}\left(\begin{array}{c}
n \\
j
\end{array}\right) E_{m, j}(y) B_{m, n-j}(x)\right) \frac{t^{n}}{n !} \\
& =\sum_{n=m}^{\infty}\left(\sum_{j=0}^{n} \frac{1}{2}\left(\begin{array}{c}
n \\
j
\end{array}\right) E_{m, j}(y) B_{m, n-j}(x)\right) \frac{t^{n}}{n !} .
\end{aligned}
$$

Here, we avoided the zero-terms on the right-hand side. Thus,

$$
\begin{aligned}
& \frac{1}{m !} \sum_{n=0}^{\infty}\left(\sum_{j=0}^{n}\left(\begin{array}{c}
n \\
j
\end{array}\right)\left(B_{m, j}(x) y^{n-j}-\frac{1}{2} E_{m, j}(y) x^{n-j}\right)\right) \frac{t^{n+m}}{n !} \\
& =\sum_{n=0}^{\infty}\left(\sum_{j=0}^{n+m} \frac{1}{2}\left(\begin{array}{c}
n+m \\
j
\end{array}\right) E_{m, j}(y) B_{m, n+m-j}(x)\right) \frac{t^{n+m}}{(n+m) !} .
\end{aligned}
$$

Comparing the coefficients on both sides, we get

$$
\begin{aligned}
& \frac{1}{m ! n !} \sum_{j=0}^{n}\left(\begin{array}{c}
n \\
j
\end{array}\right)\left(B_{m, j}(x) y^{n-j}-\frac{1}{2} E_{m, j}(y) x^{n-j}\right) \\
& =\frac{1}{(n+m) !} \sum_{j=0}^{n+m} \frac{1}{2}\left(\begin{array}{c}
n+m \\
j
\end{array}\right) E_{m, j}(y) B_{m, n+m-j}(x),
\end{aligned}
$$

from where the desired conclusion follows. 
Corollary 1. For a non-negative integer $n$, we have

$$
\begin{aligned}
& \sum_{j=0}^{n}\left(\begin{array}{l}
n \\
j
\end{array}\right) E_{j}(y)\left((x-1)^{n-j}+x^{n-j}\right)=2(x+y-1)^{n}, \\
& \sum_{j=0}^{n}\left(\begin{array}{l}
n \\
j
\end{array}\right) E_{j}(y)\left(B_{n-j}(x-1)+B_{n-j}(x)\right)=2 B_{n}(x+y-1), \\
& \sum_{j=0}^{n}\left(\begin{array}{l}
n \\
j
\end{array}\right) E_{j}(y)\left(E_{n-j}(x-1)+E_{n-j}(x)\right)=2 E_{n}(x+y-1), \\
& \sum_{j=0}^{n}\left(\begin{array}{l}
n \\
j
\end{array}\right)\left(B_{j}(x) y^{n-j}-j(y-1)^{j-1} x^{n-j}\right)=\sum_{j=0}^{n}\left(\begin{array}{l}
n \\
j
\end{array}\right)(y-1)^{j} B_{n-j}(x), \\
& \sum_{j=0}^{n}\left(\begin{array}{l}
n \\
j
\end{array}\right)\left(B_{j}(x) B_{n-j}(y)-j B_{j-1}(y-1) x^{n-j}\right)=\sum_{j=0}^{n}\left(\begin{array}{l}
n \\
j
\end{array}\right) B_{j}(y-1) B_{n-j}(x), \\
& \sum_{j=0}^{n}\left(\begin{array}{l}
n \\
j
\end{array}\right)\left(B_{j}(x) E_{n-j}(y)-j E_{j-1}(y-1) x^{n-j}\right)=\sum_{j=0}^{n}\left(\begin{array}{l}
n \\
j
\end{array}\right) E_{j}(y-1) B_{n-j}(x) \text {. }
\end{aligned}
$$

We need the following lemma to prove Corollary 1

Lemma 1. If the polynomial identity

$$
\sum_{k=0}^{n} a_{n, k}(x+\alpha)^{k}=\sum_{k=0}^{n} b_{n, k}(x+\beta)^{k}
$$

holds, then the following identities hold:

(a) the Bernoulli polynomial identity

$$
\sum_{k=0}^{n} a_{n, k} B_{k}(x+\alpha)=\sum_{k=0}^{n} b_{n, k} B_{k}(x+\beta),
$$

(b) the Euler polynomial identity

$$
\sum_{k=0}^{n} a_{n, k} E_{k}(x+\alpha)=\sum_{k=0}^{n} b_{n, k} E_{k}(x+\beta) .
$$

Proof. Affirmation (a) is Theorem 1 in [10. Affirmation (b) is obtained from (a) together with a formula expressing Euler polynomials in terms of Bernoulli polynomials. 
Proof of Corollary 1 . Since $B_{0, n}(x)=(x-1)^{n}$ and $E_{0, n}(x)=E_{n}(x)$, by setting $m=0$ in Theorem 8 , we have

$$
\sum_{j=0}^{n}\left(\begin{array}{l}
n \\
j
\end{array}\right)\left((x-1)^{j} y^{n-j}-\frac{1}{2} E_{j}(y) x^{n-j}\right)=\frac{1}{2} \sum_{j=0}^{n}\left(\begin{array}{l}
n \\
j
\end{array}\right) E_{j}(y)(x-1)^{n-j},
$$

yielding

$$
\begin{aligned}
\sum_{j=0}^{n}\left(\begin{array}{l}
n \\
j
\end{array}\right) E_{j}(y)\left((x-1)^{n-j}+x^{n-j}\right) & =2 \sum_{j=0}^{n}\left(\begin{array}{l}
n \\
j
\end{array}\right)(x-1)^{j} y^{n-j} \\
& =2(x+y-1)^{j},
\end{aligned}
$$

which is (4). The identities (5) and (6) are obtained from (4) by applying Lemma 1. On the other hand, since $B_{1, n}(x)=B_{n}(x)$ and $E_{1, n}(x)=2 n(x-1)^{n-1}$ (Theorem 10), by setting $m=1$ in Theorem 8 , we have

$$
\begin{aligned}
(n+1) \sum_{j=0}^{n}\left(\begin{array}{l}
n \\
j
\end{array}\right)\left(B_{j}(x) y^{n-j}-j(y-1)^{j-1} x^{n-j}\right) & \\
& =\sum_{j=0}^{n}\left(\begin{array}{c}
n+1 \\
n-j
\end{array}\right)(j+1)(y-1)^{j} B_{n-j}(x) .
\end{aligned}
$$

Dividing $n+1$ on both sides, we get the identity (7). The identities (8) and (9) are obtained from (7) by applying Lemma 1

Theorem 9. For non-negative integers $n$ and $m$, we have

$$
\begin{aligned}
2 \sum_{j=0}^{n}\left(\begin{array}{c}
n \\
j
\end{array}\right) E_{m+1, n-j}(x) y^{j}-\frac{2 n}{m+1} \sum_{j=0}^{n-1}\left(\begin{array}{c}
n-1 \\
j
\end{array}\right) E_{m, n-j-1}(y) x^{j} \\
=\sum_{j=0}^{n}\left(\begin{array}{c}
n \\
j
\end{array}\right) E_{m+1, n-j}(x) E_{m, j}(y) .
\end{aligned}
$$

Proof of Theorem 3. From the definition (1),

$$
\begin{aligned}
& \frac{2 t^{m+1}}{(m+1) !} e^{x t} \\
& =\left(e^{t}+1-\sum_{j=0}^{m-1} \frac{t^{j}}{j !}-\frac{t^{m}}{m !}\right) \sum_{n=0}^{\infty} E_{m+1, n}(x) \frac{t^{n}}{n !} \\
& =\left(e^{t}+1-\sum_{j=0}^{m-1} \frac{t^{j}}{j !}\right) \sum_{n=0}^{\infty} E_{m+1, n}(x) \frac{t^{n}}{n !}-\frac{t^{m}}{m !} \sum_{n=0}^{\infty} E_{m+1, n}(x) \frac{t^{n}}{n !} .
\end{aligned}
$$


By using the definition (11) again, we have

$$
\begin{aligned}
& \frac{2 t^{m+1}}{(m+1) !} e^{x t} \sum_{n=0}^{\infty} E_{m, n}(y) \frac{t^{n}}{n !} \\
& =\frac{2 t^{m}}{m !} e^{y t} \sum_{n=0}^{\infty} E_{m+1, n}(x) \frac{t^{n}}{n !}-\frac{t^{m}}{m !} \sum_{n=0}^{\infty} E_{m+1, n}(x) \frac{t^{n}}{n !} \sum_{n=0}^{\infty} E_{m, n}(y) \frac{t^{n}}{n !}
\end{aligned}
$$

or

$$
\begin{gathered}
\frac{2 n}{m+1} \sum_{n=1}^{\infty}\left(\begin{array}{c}
n-1 \\
j=0
\end{array}\left(\begin{array}{c}
n-1 \\
j
\end{array}\right) E_{m, n-j-1}(y) x^{j}\right) \frac{t^{n}}{n !} \\
=2 \sum_{n=1}^{\infty}\left(\sum_{j=0}^{n}\left(\begin{array}{c}
n \\
j
\end{array}\right) E_{m+1, n-j}(x) y^{j}\right) \frac{t^{n}}{n !} \\
\quad-\sum_{n=1}^{\infty}\left(\sum_{j=0}^{n}\left(\begin{array}{l}
n \\
j
\end{array}\right) E_{m+1, n-j}(x) E_{m, j}(y)\right) \frac{t^{n}}{n !} .
\end{gathered}
$$

Comparing the coefficients on both sides, we obtain the desired result.

Corollary 2. For a non-negative integer n, we have

$$
\begin{aligned}
& \sum_{j=0}^{n}\left(\begin{array}{l}
n \\
j
\end{array}\right)\left(2(x-1)^{n-j} y^{j}-E_{n-j}(y) x^{j}\right)=\sum_{j=0}^{n}\left(\begin{array}{c}
n \\
j
\end{array}\right)(x-1)^{n-j} E_{j}(y) \\
& \sum_{j=0}^{n}\left(\begin{array}{c}
n \\
j
\end{array}\right)\left(2 B_{n-j}(x-1) y^{j}-E_{n-j}(y) B_{j}(x)\right)=\sum_{j=0}^{n}\left(\begin{array}{c}
n \\
j
\end{array}\right) B_{n-j}(x-1) E_{j}(y), \\
& \sum_{j=0}^{n}\left(\begin{array}{c}
n \\
j
\end{array}\right)\left(2 E_{n-j}(x-1) y^{j}-E_{n-j}(y) E_{j}(x)\right)=\sum_{j=0}^{n}\left(\begin{array}{c}
n \\
j
\end{array}\right) E_{n-j}(x-1) E_{j}(y) .
\end{aligned}
$$

Proof. Since $E_{1, n}(x)=2 n(x-1)^{n-1}$ (Theorem 1), by setting $m=0$ in Theorem 3. we get (10). The identities (11) and (12) are obtained from (10) by applying Lemma 1

\section{REFERENCES}

[1] HASSEN, A.-NGUYEN, H. D.: Hypergeometric Bernoulli polynomials and Appell sequences, Int. J. Number Theory 4 (2008), 767-774.

[2] HASSEN, A.-NGUYEN, H. D.: Hypergeometric zeta functions, Int. J. Number Theory 6 (2010), 99-126. 
[3] KAMANO, K.: Sums of products of hypergeometric Bernoulli numbers, J. Number Theory 130 (2010), 2259-2271.

[4] KIM, D. S.-KIM, T.: Some identities of Frobenius-Euler polynomials arising from umbral calculus, Adv. Difference Equ. 2012 (2012), \#196.

[5] KOMATSU, T.: Hypergeometric Cauchy numbers, Int. J. Number Theory 9 (2013), 545560.

[6] KOMATSU, T.: Incomplete poly-Cauchy numbers, Monatsh. Math. 180 (2016), 271-288.

[7] KOMATSU, T.-LIPTAI, K.-MEZÖ, I.: Incomplete poly-Bernoulli numbers associated with incomplete Stirling numbers, Publ. Math. Debrecen 88 (2016), 357-368.

[8] KOMATSU, T.-MEZÖ, I.-SZALAY, L.: Incomplete Cauchy numbers, Acta Math. Hungar. 149 (2016), 306-323.

[9] SLOANE, N. J. A.: The On-Line Encyclopedia of Integer Sequences, available online at http://oeis.org

[10] PITA, C.: Carlitz-Type and Other Bernoulli Identities, J. Integer Seq. 19 (2016), Article 16.1.8.

* School of Mathematics and Statistics

WUHAN UNIVERSITY

WUHAN 430072

CHINA

E-mail address: komatsu@whu.edu.cn

** Universidad Panamericana

MeXico City

MEXICO

E-mail address: cpita@up.edu.mx 